\title{
Can Olfactory Tests Help to Diagnose Parkinson Disease?
}

\author{
Jin Kook Kim (iD \\ Department of Otorhinolaryngology-Head and Neck Surgery, Konkuk University School of Medicine, Seoul, Korea
}

Olfactory dysfunction is a common non-motor feature occurring in $75 \%-90 \%$ of Parkinson disease (PD) patients, which is unrelated to disease duration, severity, or parkinsonian medication. Accordingly, olfactory function test has been suggested as an initial screening tool for PD diagnosis in the newly published Movement Disorder Society diagnostic criteria for PD [1-3].

Although several olfactory function testing methods exist, only a few have achieved cross-cultural acceptance and are available clinically. Internationally, the most widely used tests are the University of Pennsylvania Smell Identification test (UPSIT) by Doty and the Sniffin' Sticks test developed by Kobal and colleagues. Numerous tests have been used to measure olfactory function in PD with odor identification tests being the most common; however, there are limitations to incorporating such tests into routine clinical encounter. Shorter tests would seem to be preferable from the perspective of both the patient and the neurologist, particularly within a busy clinical setting. Shorter tests have indeed been developed; among such tests is the 12-item Brief Smell Identification test (B-SIT), especially B-SIT version B for PD [1]. Numerous other brief screening tests also have been developed. These included the 3-item and 4-item Pocket Smell test series, the 3-item Quick Smell Identification test, SS-8, a 3-item version of the Sniffin' Sticks test and a 2-item version of the Open Essence Smell Identification test [1,3]. Cultural differences may have an impact on short olfactory tests to a greater extent compared to longer sets, where a greater variety of odors might balance such effects [4].

In an analysis of comparing the scores between normal and PD patients using the most commonly used identification tests which include UPSIT, Sniffin' Sticks test (SS-16), and B-SIT, the UPSIT of the PD group had a mean score of 19.4 \pm 7.2 compared to $28 \pm 8.7$ in healthy control [1]. PD group had $5.8 \pm 2.6$ correct answers in B-SIT compared to $8.8 \pm 2.7$ in control group. For SS-16, correctly identified by controls was $10.3 \pm 2.2$, while the PD group had $7.4 \pm 2.8$ correct answers. The sensitivity and specificity was $77.8 \%$ and $71.2 \%$, respectively when using a cutoff value of $\leq 9$. Lemon, turpentine, and rose had an identification rate below the 25 th percentile for all three tests. Odors with an identification rate above the 75 th percentile include banana for all three tests, and gasoline, onion, and chocolate for UPSIT and B-SIT [3]. However, these results should be interpreted considering factors such as cultural differences and individual scent familiarity. In another study using the same B-SIT, among the 12 odors, gasoline, banana, pineapple, smoke, and cinnamon were required to discriminate patients with PD from controls adequately [5]. Cao et al. [2] reported that the B-SIT score was significantly decreased in PD patients and indicated that a cutoff value of 8 , as suggested by the developers of the test, provided sensitivity of $64.1 \%$ and specificity of $83.9 \%$ for distinguishing PD patients from healthy controls. Odors such as gasoline, rubber tire, smoke, coffee, and chocolate exhibited significant differences $(P<0.01)$ in odor identification between PD and control groups. In the case of Open Essence (OE) test used in Japan, the distinction of Indian ink was effective for screening PD patients. Fujio et al. [6] suggested the discrepancy between visual analog scale and OE scores, wrong answer in Indian ink and high-selected rates of wrong alternatives other than "undetectable" in OE might be useful for differential diagnosis of PD among the elderly with olfactory disturbance [6].

In summary, evaluation of the olfactory function of suspected PD patients is valid for the diagnosis of PD, and it is especially desirable to use the low score of odor identification test. However, further research is required on evaluating the diagnostic value of detection of specific odorants.

\section{CONFLICT OF INTEREST}

No potential conflict of interest relevant to this article was reported.

Copyright () 2019 by Korean Society of Otorhinolaryngology-Head and Neck Surgery.

This is an open-access article distributed under the terms of the Creative Commons Attribution Non-Commercial License (http://creativecommons.org/licenses/by-nc/4.0)

which permits unrestricted non-commercial use, distribution, and reproduction in any medium, provided the original work is properly cited. 


\section{ORCID}

Jin Kook Kim https://orcid.org/0000-0003-4245-6252

\section{REFERENCES}

1. Morley JF, Cohen A, Silveira-Moriyama L, Lees AJ, Williams DR, Katzenschlager R, et al. Optimizing olfactory testing for the diagnosis of Parkinson's disease: item analysis of the University of Pennsylvania smell identification test. NPJ Parkinsons Dis. 2018 Jan;4:2.

2. Cao M, Li Y, Gu Z, Mi T, Xu X, Ma C, et al.Validation of the utility of the brief smell identification test in Chinese patients with Parkinson's disease. J Clin Neurosci. 2019 Feb;60:68-72.

3. Rodriguez-Violante M, Gonzalez-Latapi P, Camacho-Ordonez A, Martinez-Ramirez D, Morales-Briceno H, Cervantes-Arriaga A. Com- paring the accuracy of different smell identification tests in Parkinson's disease: relevance of cultural aspects. Clin Neurol Neurosurg. 2014 Aug;123:9-14.

4. Mahlknecht P, Pechlaner R, Boesveldt S, Volc D, Pinter B, Reiter E, et al. Optimizing odor identification testing as quick and accurate diagnostic tool for Parkinson's disease. Mov Disord. 2016 Sep;31(9): 1408-13.

5. Double KL, Rowe DB, Hayes M, Chan DK, Blackie J, Corbett A, et al. Identifying the pattern of olfactory deficits in Parkinson disease using the brief smell identification test. Arch Neurol. 2003 Apr;60(4): 545-9.

6. Fujio H, Inokuchi G, Tatehara S, Kuroki S, Fukuda Y, Kowa H, et al. Characteristics of smell identification test in patients with Parkinson disease. Clin Exp Otorhinolaryngol. 2019 May;12(2):206-11.

Received February 6, 2019 Accepted February 27, 2019 